\title{
RESEARCH
}

Open Access

\section{Deciding the operation type according to mismatch repair status among hereditary nonpolyposis colorectal cancer patients: should a tailored approach be applied, or does one size fit all?}

\author{
Chun-Kai Liao', Yueh-Chen Lin', Yu-Jen Hsu', Yih-Jong Chern'1, Jeng-Fu You' and Jy-Ming Chiang ${ }^{1,2^{*}}$ (i)
}

\begin{abstract}
Background: Although extended colectomy (EC) was recommended for HNPCC patients, previous studies did not show significantly improved overall survival. Immunohistochemical (IHC) stain of mismatch repair (MMR) gene protein expression is now a feasible and reliable test clinically. Therefore, we tried to investigate whether we could use MMR IHC stain to select operation types in HNPCC patients.

Patients and methods: Between 1995 and 2013, 186 HNPCC patients were collected. Status of MMR protein expression, perioperative clinic-pathological variables and post-operative follow up status were analyzed by multivariate analyses.
\end{abstract}

Results: Sixty-five percent (121 of 186) patients of these HNPCC patients demonstrated loss of at least one MMR protein. There were several significant differences existing between deficient MMR (dMMR) and proficient MMR (pMMR) subgroups in terms of clinic-pathological characteristics. With the average follow-up duration of 93.9 months, we observed significantly high risk of developing metachronous CRC between SC and EC subgroups (crude rate $8.5 \%$ vs. $0 \%, p=0.035$ ). However, no significant difference was observed among the presence of extracolonic tumors $(12.4 \%$ vs. $5.8 \%, p=0.284)$. The positive and negative prediction rate of metachronous CRC in dMMR subgroup was 12.8 and $87.2 \%$ while 1.9 and $98.1 \%$ in the pMMR subgroup.

Survival outcomes were significantly affected by MMR status and resection types by multivariate analysis. Significantly better $O S$ in $\mathrm{dMMR}$ subgroup ( $\mathrm{HR}=0.479,95 \% \mathrm{Cl}: 0.257-0.894, p=0.021)$ comparing with pMMR subgroup was observed. However, significant improved DFS (HR $=0.367,95 \% \mathrm{Cl}: 0.172-.0787, p=0.010)$ but not significant for OS ( $H R=0.510,95 \% \mathrm{Cl}: 0.219-1.150, p=0.103)$ for EC subgroup compared with SC subgroup.

Differences existing among different subgroups by combing extent of resection and MMR status. In dMMR subgroup, SC, compared with EC, demonstrated significantly worse DFS by multivariate analyses (HR $=3.526$, 95\% Cl: 1.346-9.236, $p=0.010$ ) but not for OS ( $\mathrm{HR}=2.387,95 \% \mathrm{Cl}: 0.788-7.229, p=0.124)$, however, no

\footnotetext{
*Correspondence: jmjiang@cgmh.adm.tw; jmjiang1234@yahoo.com.tw

'Colorectal Section, Department of Surgery, Chang Gung Memorial Hospital, No. 5, Fuxing St., Guishan Dist, Taoyuan, Taiwan 33305

${ }^{2}$ College of Medicine, Chang Gung University, Taoyuan, Taiwan
}

(c) The Author(s). 2021 Open Access This article is licensed under a Creative Commons Attribution 4.0 International License, which permits use, sharing, adaptation, distribution and reproduction in any medium or format, as long as you give appropriate credit to the original author(s) and the source, provide a link to the Creative Commons licence, and indicate if changes were made. The images or other third party material in this article are included in the article's Creative Commons licence, unless indicated otherwise in a credit line to the material. If material is not included in the article's Creative Commons licence and your intended use is not permitted by statutory regulation or exceeds the permitted use, you will need to obtain permission directly from the copyright holder. To view a copy of this licence, visit http://creativecommons.org/licenses/by/4.0/. The Creative Commons Public Domain Dedication waiver (http://creativecommons.org/publicdomain/zero/1.0/) applies to the data made available in this article, unless otherwise stated in a credit line to the data. 
significantly differences of OS and DFS in PMMR subgroup between SC and EC were found.

Conclusions: Significantly better overall survival and higher rate of metachronous CRC exist in dMMR subgroup of HNPCC patients comparing with pMMR subgroup. Extended colectomy significantly improved DFS and was thus recommended for dMMR subgroup but not pMMR subgroup of HNPCC patients.

Keywords: HNPCC, MMR status, Extended colectomy, Metachronous CRC, Overall survival

\section{Background}

Hereditary nonpolyposis colorectal cancer (HNPCC) patients defined by Amsterdam criteria clinically. These patients were characterized with increased risks of metachronous colorectal tumors [1] and extra-colonic cancers $[2,3]$, in addition to younger age at onset, right side colon predominance, higher proportion of poorly differentiated and mucinous adenocarcinoma $[2,4,5]$.

To improve surgical outcomes of HNPCC patients, extended colectomy (EC), such as subtotal or total colectomy, rather than segmental colectomy (SC) or hemicolectomy, is recommended owing to the nature of increased risks of metachronous colorectal tumors among HNPCC patients [6-11]. Following these recommended guidelines has showed to decrease the rate of metachronous colorectal lesions significantly [1, 6-11], however, the survival rate seems not significantly improved for extended colectomy subgroups [7, 11, 12]. The reasons may be resulted from adequate post colectomy colonoscopy surveillance or these cancers are detected at an early stage. In contrast, functional results and quality of life post extended colectomy (EC) was concerned with frequency, urgency and varied degrees of stool incontinence [13]. Therefore, the optimal operation type among HNPCC patients remains controversial.

Universal testing of all newly diagnosed CRC patients was considered to be practicable currently and tested for deficient mismatch repair (dMMR) protein expression status may be useful in clinical practice [14] such as to determine post-operative adjuvant chemotherapy for stage III patients [15] or identification of Lynch syndrome patients from familial colorectal cancer type $\mathrm{X}$ (FCCTX) patients that have distinct clinical behavior form [16-18]. However, the value of pre-colectomy status of MMR expression related to surgical treatment of HNPCC patients remained unclear. In this retrospective study, we analyzed 186 HNPCC patients to investigate whether MMR expression status may help decide types of colectomy among these patients and to achieve improved overall and disease-free survival.

\section{Material and methods Patient selection}

Between January 1995 and September 2013, a total of 186 consecutive patients fulfilled the Amsterdam criteria
II (AC II) underwent colectomy for colorectal cancer at Chang Gung Memorial Hospital (CGMH) were retrospectively analyzed. Patients fulfilling the AC II (at least three relatives with a Lynch-associated cancer, one being a first-degree relative of the other two; at least two successive generations affected; and at least one person diagnosed before 50 years of age) were defined as HNPCC patients. The study was approved by the IRB of Chang Gung Memorial Hospital (IRB201801201B).

\section{Data collection}

A retrospective analysis of data from the Colorectal Section Tumor Registry in Chang Gung Memorial Hospital, a prospectively designed database consisting of the records of postoperative patients who were consecutively and actively followed up, was conducted. Written informed consent was obtained from the patients prior to study participation. This study was approved by the Institutional Review Board of Chang Gung Memorial Hospital (approval number IRB102-2284B), and performed in accordance with the Declaration of Helsinki. All data were recorded in the hospital database and used for research purposes.

The peri-operative variables including age, sex, tumor location and surgery type were analyzed. The pathological parameters including MMR status, T-stage, Nstage, M-stage, histology grade and histology type were also analyzed.

\section{IHC analysis for MMR gene expression}

Paraffin-embedded tumor blocks from HNPCC patients were retrieved from the Pathology Department of CGMH. For each patient, 4- $\mu \mathrm{m}$ thick sections from one formalin-fixed, paraffin-embedded tissue block containing both tumor tissue and normal adjacent mucosa were obtained. Immunostaining was performed on a Dako Universal Autostainer (DakoCytomation, Denmark) by using ChemMateTM EnvisonTM + Detection kits (DakoCytomation, Denmark) and described before [19]. For the evaluation of IHC results, abnormal staining was defined as total loss of protein in the tumor, using appropriate controls; staining was considered assessable when the nucleus was stained in cells serving as internal controls, including either stromal or germinal follicle lymphocytes or normal epithelial cells in the crypt bases. 
Tumors were considered negative for MMR protein expression when neoplastic cells showed complete absence of detectable nuclear staining in a sample for which internal positive controls were stained. Pathologists who had no knowledge of the family history or other clinicopathological features, reviewed all cases to confirm the immunostaining results.

\section{Follow up}

All physicians at the author's institution adopted similar follow-up routines and adjuvant treatment protocols. After primary tumor resection, all patients were subjected to a follow-up program that included outpatient visits every 3 to 6 months for physical examinations and carcinoembryonic antigen (CEA) tests. Chest radiography, abdominal ultrasonography, or abdominal computed tomography (CT) imaging, in addition to colonoscopy, were performed one year after the index surgery and then every 1 to 3 years whenever necessary. Follow-up status was confirmed postoperatively every 12 months by a team of three physicians and five specially trained nurses. Telephone interview or mail questionnaire was made if patient's medical records were not available. Date of first recurrence was defined as the first date when the existence of local recurrence and/or distant metastases was confirmed by histology of biopsy specimens, additional surgery, and/or by radiological studies. The last follow-up date in this study was May 31, 2020.

\section{Statistical analyses}

All parameters were analyzed using the Statistical Package for Social Sciences (SPSS) version 26 (IBM Corp., Armonk, New York). The categorical variables were compared using Pearson's chi-squared test or Fisher's exact test, whereas continuous variables were compared using the independent sample T-test. Survival analysis was performed using Kaplan-Meier curves with the logrank test. The Cox proportional hazards model was used to investigate the effect of clinical variables on survival while adjusting for other explanatory variables. The $p$ values were two-sided and those $<0.05$ were considered statistically significant.

\section{Results}

In this cohort, a total of 186 patients fulfilled with Amsterdam criteria II were included. As shown in Table 1, Sixty-five percent (121 of 186) patients of these HNPCC patients demonstrated loss of at least one MMR protein. The distribution of the loss of MMR gene expression (shown in Table 2) included concordant losses of MLH1/PMS2 staining (82 of 121 patients, 67.8\%), MSH2/MSH6 staining (35 of 121 patients, $28.9 \%$ ) and loss of PMS2 only (4 of 121 patients, 3.3\%).
Table 1 Basic characteristics and clinicopathological features among 186 HNPCC patients

\begin{tabular}{|c|c|c|c|}
\hline & dMMR $(n=121)$ & pMMR $(n=65)$ & $p$ Value \\
\hline Age (mean $\pm S D)$, years & $47.54 \pm 12.39$ & $54.62 \pm 13.67$ & $<0.001$ \\
\hline \multicolumn{4}{|l|}{ Sex } \\
\hline Male & $59(48.8)$ & $34(52.3)$ & \multirow[t]{2}{*}{0.645} \\
\hline Female & $62(51.2)$ & $31(47.7)$ & \\
\hline \multicolumn{4}{|l|}{ Cancer location } \\
\hline Right colon & $74(61.2)$ & $22(33.8)$ & \multirow[t]{3}{*}{$<0.001$} \\
\hline Left colon & $30(24.8)$ & $20(30.8)$ & \\
\hline Rectum & $17(14.0)$ & $23(35.4)$ & \\
\hline \multicolumn{4}{|l|}{ Histological type } \\
\hline Adenocarcinoma & $94(77.7)$ & $87(87.7)$ & \multirow[t]{5}{*}{$0.058^{*}$} \\
\hline Signet ring cell & $2(1.7)$ & $2(3.1)$ & \\
\hline Mucinous & $22(18.2)$ & $5(7.7)$ & \\
\hline NET & $0(0)$ & $1(1.5)$ & \\
\hline Other & $3(2.5)$ & $0(0)$ & \\
\hline \multicolumn{4}{|l|}{ Histological grade } \\
\hline Grade I & $17(14)$ & $10(15.4)$ & \multirow[t]{4}{*}{$0.048^{*}$} \\
\hline Grade II & $70(57.9)$ & $47(72.3)$ & \\
\hline Grade III/IV & $31(25.6)$ & $8(12.3)$ & \\
\hline Unclassified & $3(2.5)$ & $0(0)$ & \\
\hline \multicolumn{4}{|l|}{ Operation type } \\
\hline Segmental resection & $78(64.5)$ & $51(78.5)$ & \multirow[t]{3}{*}{$0.021^{*}$} \\
\hline Extended resection & $41(33.9)$ & $11(16.9)$ & \\
\hline Local resection & $2(1.7)$ & $3(4.6)$ & \\
\hline \multicolumn{4}{|l|}{ T stage } \\
\hline T0 & $2(1.7)$ & $1(1.6)$ & \multirow[t]{5}{*}{0.397} \\
\hline $\mathrm{T} 1$ & $6(5.0)$ & $5(7.8)$ & \\
\hline $\mathrm{T} 2$ & $10(8.3)$ & $4(6.3)$ & \\
\hline T3 & $52(43.0)$ & $35(54.7)$ & \\
\hline T4 & $51(42.1)$ & 19 (29.7) & \\
\hline \multicolumn{4}{|l|}{ N stage } \\
\hline NO & 83 (68.6) & $32(50)$ & \multirow[t]{3}{*}{0.013} \\
\hline N1 & $29(24.0)$ & 19 (29.7) & \\
\hline $\mathrm{N} 2$ & $9(7.4)$ & $13(20.3)$ & \\
\hline \multicolumn{4}{|l|}{ M stage } \\
\hline Mo & $10(8.3)$ & $6(9.2)$ & \multirow[t]{2}{*}{0.823} \\
\hline M1 & $111(91.7)$ & $59(90.8)$ & \\
\hline
\end{tabular}

*Fishers exact test

As shown in Table 1, there were several significant differences existing between dMMR and pMMR subgroups in terms of clinic-pathological characteristics. The mean age of diagnosis is younger in dMMR subgroup (47.5 vs. 54.6 years-old, $p<0.001)$. There is more extended colectomy in dMMR subgroup (Extended resection: $33.9 \%$ vs. $16.9 \%, p=0.021)$. dMMR subgroup also presented with 
Table 2 Patterns of gene loss among 186 HNPCC patients

\begin{tabular}{lll}
\hline IHC staining pattern & $\mathbf{n}$ & Percentage \\
\hline Absence of MLH1/PMS2 & 82 & $44.10 \%$ \\
Absence of MSH2/MSH6 & 35 & $18.80 \%$ \\
Absence of PMS2 & 4 & $2.20 \%$ \\
No loss & 65 & $34.90 \%$ \\
\hline
\end{tabular}

right colon predominance (right-sided colon: $61.2 \%$ vs. $33.8 \%, \mathrm{p}<0.001$ ) and more N0 stage (N0 stage: $68.6 \%$ vs. $50.0 \%, p=0.013)$.

The follow up status was summarized in Table 3 . The average follow-up duration for total patients were 93.9 months, with 111.1 and 88.5 months in EC subgroup and SC subgroups respectively. In this cohort, we observed significantly high risk of developing metachronous CRC between SC and EC subgroups (crude rate $8.5 \%$ vs. $0 \%, p=0.035$ ). Time to developing metachronous CRC was average 39.6 months (range from 4.7 to 99.3 months). However, no significant difference was observed among the presence of extra-colonic tumors $(12.4 \%$ vs. $5.8 \%, p=0.284)$.
Clinico-pathological factors related to OS (Table 4) and DFS (Table 5) were analyzed by univariate and multivariate analysis after excluding 5 patients without curative resection. There were some significant factors existed relating to $O S$ and DFS. As shown in Table 4 and Table 5 , in univariate analysis, MMR status showing a significantly better OS $(p=0.002)$ (Fig. 1A) but not DFS $(p=0.08)$ (Fig. 1B) for dMMR subgroup comparing with pMMR subgroup was observed. Multivariate analysis further confirmed significantly better OS in dMMR subgroup ( $\mathrm{HR}=0.479,95 \% \mathrm{CI}: 0.257-0.894, p=0.021$ ). Resection types also impact on surgical outcomes. Univariate analysis showed significantly better OS $(p=$ 0.003) (Fig. 2A) and DFS $(p<0.001)$ (Fig. 2B) for EC subgroup comparing with SC subgroup. Multivariate analysis further confirmed significant DFS $(\mathrm{HR}=0.367$, 95\% CI: $0.172-.0787, p=0.010$ ) but not significant for OS (HR $=0.510,95 \% \mathrm{CI}: 0.219-1.150, p=0.103)$ for $\mathrm{EC}$ subgroup compared with SC subgroup.

We further analyzed if significant surgical outcomes exist by combing these two factors interaction (extent of resection and MMR status). Showing as Fig. 3A and B,

Table 3 Survival data of 181 HNPCC patients who underwent surgical resection of CRC

\begin{tabular}{|c|c|c|c|}
\hline & Segmental resection $(n=129)$ & Extended resection $(n=52)$ & p Value \\
\hline \multicolumn{4}{|c|}{ Follow-up time, months } \\
\hline Mean & $88.50 \pm 43.92$ & $111.10 \pm 25.29$ & \multirow[t]{2}{*}{0.001} \\
\hline Median & $119.54(0.95-143.64)$ & 121.05 (31.34-133.78) & \\
\hline \multicolumn{4}{|c|}{ Survival, n (\%) } \\
\hline Dead & $45(34.9)$ & $7(13.5)$ & \multirow[t]{2}{*}{0.004} \\
\hline Alive & $84(65.1)$ & $45(86.5)$ & \\
\hline \multicolumn{4}{|l|}{ Recurrence } \\
\hline Presence & $24(18.6)$ & $4(7.7)$ & \multirow[t]{2}{*}{0.073} \\
\hline Absence & $105(81.4)$ & $48(92.3)$ & \\
\hline \multicolumn{4}{|c|}{ Recurrence interval, months } \\
\hline Mean & $18.66 \pm 14.12$ & $49.29 \pm 21.76$ & \multirow[t]{2}{*}{0.001} \\
\hline Median & $11.86(3.68-67.58)$ & $54.8(20.01-67.58)$ & \\
\hline \multicolumn{4}{|c|}{ Metachronous colon lesions } \\
\hline Presence & $11(8.5)$ & $0(0)$ & \multirow[t]{2}{*}{$0.035^{*}$} \\
\hline Absence & $118(91.5)$ & $52(100)$ & \\
\hline \multicolumn{4}{|c|}{ Recurrence interval, months } \\
\hline Mean & $39.64 \pm 30.45$ & & \\
\hline Median & 34.07 (4.70-99.35) & & \\
\hline \multicolumn{4}{|c|}{ Extracolonic lesion } \\
\hline Presence & $16(12.4)$ & $3(5.8)$ & \multirow[t]{2}{*}{0.284} \\
\hline Absence & $113(87.6)$ & $49(94.2)$ & \\
\hline \multicolumn{4}{|c|}{ Recurrence interval, months } \\
\hline Mean & $57.70 \pm 41.60$ & $76.16 \pm 47.20$ & \multirow[t]{2}{*}{0.497} \\
\hline Median & $51.78(46.13-130.56)$ & $30.62(16.92-130.56)$ & \\
\hline
\end{tabular}


Table 4 Univariate and multivariate analyses of clinicopathological features with OS among 181 HNPCC patients who underwent curative resection of $C R C$

\begin{tabular}{|c|c|c|c|c|c|c|c|c|}
\hline \multirow[t]{2}{*}{ Characteristics } & \multirow[t]{2}{*}{$n$} & \multicolumn{3}{|l|}{ OS (\%) } & \multirow{2}{*}{$\begin{array}{l}\mathrm{p} \\
\text { Value }\end{array}$} & \multicolumn{3}{|c|}{ Multiple Cox } \\
\hline & & 1 year & 3 years & 5 years & & $\mathrm{HR}$ & $95 \% \mathrm{Cl}$ & p Value \\
\hline \multicolumn{9}{|l|}{ Age, years } \\
\hline$<40$ & 42 & 95.2 & 90.3 & 90.3 & 0.05 & 1 & & \\
\hline$\geq 40$ & 139 & 95 & 83.4 & 78.3 & & 2.176 & $0.933-5.075$ & 0.072 \\
\hline \multicolumn{9}{|l|}{ Sex } \\
\hline Male & 90 & 94.5 & 85.6 & 81.2 & 0.688 & & & \\
\hline Female & 91 & 95.5 & 84.3 & 80.9 & & & & \\
\hline \multicolumn{9}{|l|}{ MMR status } \\
\hline $\mathrm{pMMR}$ & 62 & 95.2 & 77.4 & 71 & 0.002 & 1 & & \\
\hline $\mathrm{dMMR}$ & 119 & 95 & 89 & 86.4 & & 0.479 & $0.257-0.894$ & 0.021 \\
\hline \multicolumn{9}{|l|}{ Tumor location } \\
\hline Right colon & 94 & 95.7 & 88.1 & 86 & 0.001 & 1 & & \\
\hline Left colon & 50 & 98 & 92 & 90 & & 1.254 & $0.620-2.537$ & 0.53 \\
\hline Rectum & 37 & 89.2 & 67.6 & 56.8 & & 1.302 & $0.628-2.702$ & 0.478 \\
\hline \multicolumn{9}{|l|}{ Operation type } \\
\hline Segmental resection & 129 & 93 & 79.6 & 74.9 & 0.003 & 1 & & \\
\hline Extended resection & 52 & 100 & 98.1 & 96.2 & & 0.501 & $0.219-1.150$ & 0.103 \\
\hline \multicolumn{9}{|l|}{ Histological type } \\
\hline Adenocarcinoma & 146 & 95.9 & 84.8 & 79.9 & 0.993 & & & \\
\hline Signet ring cell/mucinous & 31 & 93.5 & 87.1 & 87.1 & & & & \\
\hline Other & 4 & 75 & 75 & 75 & & & & \\
\hline \multicolumn{9}{|l|}{ Histological grade } \\
\hline Grade I & 25 & 100 & 100 & 92 & 0.509 & & & \\
\hline Grade II & 116 & 94 & 82.6 & 78.3 & & & & \\
\hline Grade III/IV & 38 & 94.7 & 81.2 & 81.2 & & & & \\
\hline Unclassified & 2 & 100 & 100 & 100 & & & & \\
\hline \multicolumn{9}{|l|}{ T stage } \\
\hline T0 & 3 & 100 & 100 & 100 & 0.073 & & & \\
\hline $\mathrm{T} 1$ & 10 & 100 & 100 & 80 & & & & \\
\hline $\mathrm{T} 2$ & 14 & 92.9 & 92.9 & 92.9 & & & & \\
\hline $\mathrm{T} 3$ & 86 & 98.8 & 84.5 & 82.1 & & & & \\
\hline $\mathrm{T} 4$ & 68 & 89.7 & 80.9 & 76.5 & & & & \\
\hline \multicolumn{9}{|l|}{$N$ stage } \\
\hline NO & 112 & 97.3 & 93.8 & 90.2 & $<0.001$ & 1 & & \\
\hline N1 & 48 & 93.7 & 78.5 & 74.1 & & 1.343 & $0.655-2.756$ & 0.421 \\
\hline N2 & 21 & 85.7 & 52.4 & 47.6 & & 3.605 & $1.623-8.009$ & 0.002 \\
\hline \multicolumn{9}{|l|}{ M stage } \\
\hline MO & 167 & 97.6 & 89.1 & 86.1 & $<0.001$ & 1 & & \\
\hline M1 & 14 & 64.3 & 35.7 & 19 & & 7.723 & $3.362-17.742$ & $<0.001$ \\
\hline
\end{tabular}

there was significantly difference in terms of OS $(p=$ $0.001)$ and DFS $(p=0.002)$ existing among different subgroups after combing extent of resection and MMR status (Table 6). dMMR plus extended resection showed best survival benefit in both OS and DFS while pMMR with segmental resection had worst survival (Table 6). We then analyzed dMMR and pMMR subgroups respectively, as shown in Fig. 4A and B. Shown in Table 7, 
Table 5 Univariate and multivariate analyses of clinicopathological features with DFS among 181 HNPCC patients who underwent curative resection for colorectal cancer

\begin{tabular}{|c|c|c|c|c|c|c|c|c|}
\hline \multirow[t]{2}{*}{ Characteristics } & \multirow[t]{2}{*}{$\mathrm{n}$} & \multicolumn{3}{|c|}{ DFS (\%) } & \multirow{2}{*}{$\begin{array}{l}p \\
\text { Value }\end{array}$} & \multicolumn{3}{|c|}{ Multiple Cox } \\
\hline & & 1 year & 3 years & 5 years & & HR & $95 \% \mathrm{Cl}$ & p Value \\
\hline \multicolumn{9}{|l|}{ Age, years } \\
\hline$<40$ & 42 & 95.2 & 90.3 & 87.9 & 0.081 & & & \\
\hline$\geq 40$ & 139 & 92.1 & 79 & 73.9 & & & & \\
\hline \multicolumn{9}{|l|}{ Sex } \\
\hline Male & 90 & 92.2 & 79.8 & 76.4 & 0.967 & & & \\
\hline Female & 91 & 93.4 & 83.4 & 77.8 & & & & \\
\hline \multicolumn{9}{|l|}{ MMR status } \\
\hline $\mathrm{pMMR}$ & 62 & 95.2 & 77.4 & 71 & 0.08 & & & \\
\hline $\mathrm{dMMR}$ & 119 & 91.6 & 83.9 & 80.5 & & & & \\
\hline \multicolumn{9}{|l|}{ Tumor location } \\
\hline Right colon & 94 & 94.7 & 84.9 & 82.7 & 0.02 & 1 & & \\
\hline Left colon & 50 & 94 & 88 & 84 & & 1.077 & $0.582-1.992$ & 0.813 \\
\hline Rectum & 37 & 86.5 & 64.9 & 54.1 & & 1.546 & $0.837-2.854$ & 0.164 \\
\hline \multicolumn{9}{|l|}{ Operation type } \\
\hline Segmental resection & 129 & 90.7 & 75.7 & 71 & $<0.001$ & 1 & & \\
\hline Extended resection & 52 & 98.1 & 96.2 & 92.3 & & 0.367 & $0.172-0.787$ & 0.01 \\
\hline \multicolumn{9}{|l|}{ Histological type } \\
\hline Adenocarcinoma & 146 & 93.2 & 80.6 & 75.1 & 0.812 & & & \\
\hline Signet ring cell/mucinous & 31 & 93.5 & 87.1 & 87.1 & & & & \\
\hline Other & 4 & 75 & 75 & 75 & & & & \\
\hline \multicolumn{9}{|l|}{ Histological grade } \\
\hline Grade I & 25 & 100 & 96 & 2 & 0.164 & & & \\
\hline Grade II & 116 & 92.2 & 80.1 & 74 & & & & \\
\hline Grade III/IV & 38 & 89.5 & 75.9 & 75.9 & & & & \\
\hline Unclassified & 2 & 100 & 100 & 100 & & & & \\
\hline \multicolumn{9}{|l|}{ T stage } \\
\hline T0 & 3 & 100 & 100 & 100 & 0.056 & & & \\
\hline $\mathrm{T} 1$ & 10 & 100 & 100 & 80 & & & & \\
\hline $\mathrm{T} 2$ & 14 & 92.9 & 92.9 & 92.9 & & & & \\
\hline $\mathrm{T} 3$ & 86 & 97.7 & 82.2 & 79.8 & & & & \\
\hline $\mathrm{T} 4$ & 68 & 85.3 & 75 & 69.1 & & & & \\
\hline \multicolumn{9}{|l|}{ N stage } \\
\hline NO & 112 & 94.6 & 89.3 & 84.8 & $<0.001$ & 1 & & \\
\hline N1 & 48 & 91.7 & 76.4 & 72 & & 1.521 & $0.839-2.759$ & 0.167 \\
\hline N2 & 21 & 85.7 & 52.4 & 47.6 & & 2.715 & $1.286-5.732$ & 0.009 \\
\hline \multicolumn{9}{|l|}{ M stage } \\
\hline MO & 167 & 95.2 & 85.5 & 81.9 & $<0.001$ & 1 & & \\
\hline M1 & 14 & 64.3 & 35.7 & 19 & & 4.231 & $2.013-8.889$ & $<0.001$ \\
\hline
\end{tabular}


a

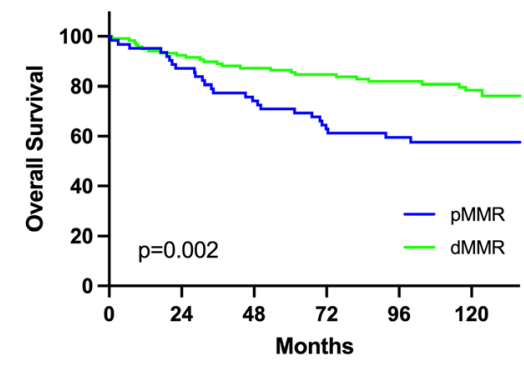

Number at risk

$\begin{array}{lllllll}\text { dMMR } & 119 & 109 & 103 & 97 & 81 & 61\end{array}$ b

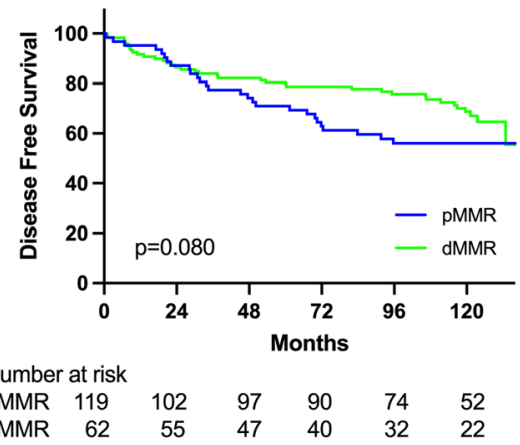

Fig. 1 Comparison of overall survival (a) and disease-free survival (b) between patients with proficient mismatch repair gene (pMMR) and those with deficient mismatch repair gene (dMMR)

in dMMR subgroup, SC, compared with EC, demonstrated significantly worse DFS by multivariate analyses $(\mathrm{HR}=3.526,95 \% \mathrm{CI}: 1.346-9.236, p=0.010)$ but not significantly difference for OS ( $\mathrm{HR}=2.387,95 \%$ CI: 0.788 7.229, $p=0.124$ ), however, no significantly differences of OS and DFS in pMMR subgroup between SC and EC were found.

In our cohort, there were no metachronous CRC for patients underwent EC during follow up period. However, 11 patients developing metachronous CRC in the SC subgroup (median: 34.1 months, ranging from 4.7 to 99.3 months), the positive and negative prediction rate of metachronous CRC in AMMR subgroup was 12.8 and $87.2 \%$ while 1.9 and $98.1 \%$ in the pMMR subgroup.

\section{Discussion}

In this retrospective study, we demonstrated that among clinically defined HNPCC patients, by multivariate analyses, extended colectomy significantly improved disease free survival but no overall survival improvement compared with segmental colectomy for dMMR patients. In contrast, there is no significant difference for both DFS and OS among pMMR patients.

Previous studies recommended that EC rather than SC for the index tumor management, which become the choice of surgical treatment for LS patients because of its high risk of metachronous CRC [1, 5-11]. However, merit of EC compared with SC among clinical defined HNPCC patients became complex because HNPCC was a more heterogeneous group [20]. At least, two subgroups (dMMR and pMMR) exist in the HNPCC patients $[16-18,20]$. Significantly lower risk of patients developing metachronous colorectal cancer in pMMR subgroup had been reported compared with dMMR subgroup $[4,16-18,20]$. In this study, we demonstrated EC significantly affect disease free survival in the dMMR subgroup (HR $=0.284,95 \% \mathrm{CI}: 0.108-.0743, p=0.010$, Fig. 4B). However, EC did not impact on DFS comparing with SC in the pMMR subgroup (Fig. 4D). Data from the present study reflected that the risk of metachronous colorectal cancer of pMMR subgroup was significantly lower than dMMR subgroup after SC (in this study, $12.8 \%$ dMMR patients in contrast to $1.9 \%$ pMMR
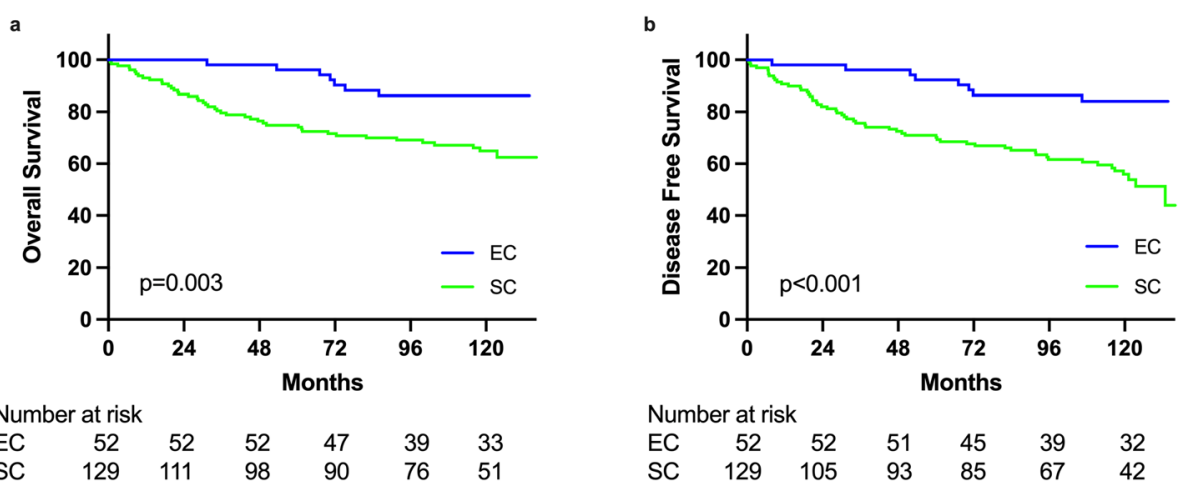

Fig. 2 Comparison of overall survival (a) and disease-free survival (b) between patients who had extended colectomy (EC) and those who had segmental colectomy (SC) 

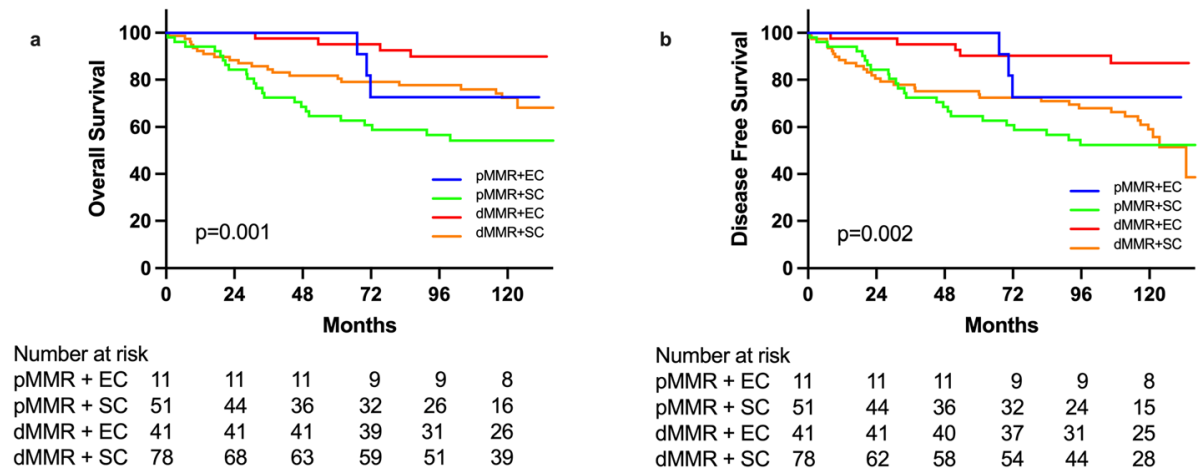

Fig. 3 Comparison of overall survival (a) and disease-free survival (b) in HNPCC patients by combination of mismatch repair gene type and surgery type

patient underwent SC developed metachronous CRC). These findings suggested that MMR status may be considered as an important surrogate factor in HNPCC patients for making decision of operation types.

Regarding index tumor located at rectum, should we do proctocolectomy with ileoanal reservoir or low anterior resection become another question. Most surgeons would not take it lightly but they still worried about second CRC after longer follow up years. In this study, we found 40 patients with rectal cancer diagnosed, 23 (57.5\%) are pMMR while 17 (42.5\%) are dMMR cases. Among these patients, 3 patients developed metachronous CRC and they were all dMMR subtype of index cancer. We may well address that proctocolectomy can be considered only if rectal cancer with dMMR status was confirmed.
Although in the era of genomics, sophisticated development of genetic test makes it feasible in clinical practice, some patients still refuse to undergo genetic test or cannot afford these tests in regions without health insurance reimbursement such as in Taiwan. In this case, family history (AC-II) combined with immunohistochemistry test of MMR proteins provide a very good surrogate in practice in the real world. Preoperative testing with MMR status is important not only because it can affect surgical decision making but also its relatively cost effect benefit in clinical practice. MMR status could provide information similar to genetic data. According to a report by Quezada-Diaz et al. [21], in Lynch syndrome patients, MSH6 or PMS2 pathogenic variant could be recommended with SC and long-term colonoscopy surveillance rather than $\mathrm{EC}$ because no metachronous CRC detected during a 10-year

Table 6 Univariate and multivariate analyses of clinicopathological features with OS and DFS among 181 HNPCC patients who underwent curative resection of CRC

\begin{tabular}{|c|c|c|c|c|c|c|c|c|}
\hline \multirow[t]{2}{*}{ Characteristics } & \multirow[t]{2}{*}{$\mathrm{n}$} & \multicolumn{3}{|l|}{ OS (\%) } & \multirow{2}{*}{$\begin{array}{l}\mathrm{p} \\
\text { Value }\end{array}$} & \multicolumn{3}{|c|}{ Multiple Cox* } \\
\hline & & 1 year & 3 years & 5 years & & HR & $95 \% \mathrm{Cl}$ & $\mathrm{p}$ Value \\
\hline \multicolumn{9}{|c|}{ MMR with operation type } \\
\hline $\mathrm{pMMR+EC}$ & 11 & 100 & 100 & 100 & 0.001 & 1 & & \\
\hline $\mathrm{pMMR}+\mathrm{SC}$ & 51 & 94.1 & 72.5 & 64.7 & & 1.906 & $0.548-6.629$ & 0.31 \\
\hline $\mathrm{dMMR+EC}$ & 41 & 100 & 97.6 & 95.1 & & 0.447 & $0.095-2.103$ & 0.308 \\
\hline $\mathrm{dMMR}+\mathrm{SC}$ & 78 & 92.3 & 84.4 & 81.8 & & 0.924 & $0.256-3.326$ & 0.903 \\
\hline \multirow[t]{2}{*}{ Characteristics } & $\mathrm{n}$ & \multicolumn{3}{|l|}{ DFS (\%) } & p Value & \multicolumn{3}{|c|}{ Multiple Cox** } \\
\hline & & 1 year & 3 years & 5 years & & $H R$ & $95 \% \mathrm{Cl}$ & $\mathrm{p}$ Value \\
\hline \multicolumn{9}{|c|}{ MMR with operation type } \\
\hline $\mathrm{pMMR+EC}$ & 11 & 100 & 100 & 100 & 0.002 & 1 & & \\
\hline $\mathrm{pMMR}+\mathrm{SC}$ & 51 & 94.1 & 72.5 & 64.7 & & 1.814 & $0.530-6.207$ & 0.343 \\
\hline $\mathrm{dMMR+EC}$ & 41 & 97.6 & 95.1 & 90.2 & & 0.467 & $0.108-2.026$ & 0.309 \\
\hline $\mathrm{dMMR}+\mathrm{SC}$ & 78 & 88.4 & 77.9 & 75.3 & & 1.476 & $0.434-5.017$ & 0.532 \\
\hline
\end{tabular}

${ }^{*}$ Confounding with age, tumor location, $\mathrm{N}$ stage, and $\mathrm{M}$ stage

**Confounding with tumor location, $\mathrm{N}$ stage, and $\mathrm{M}$ stage 

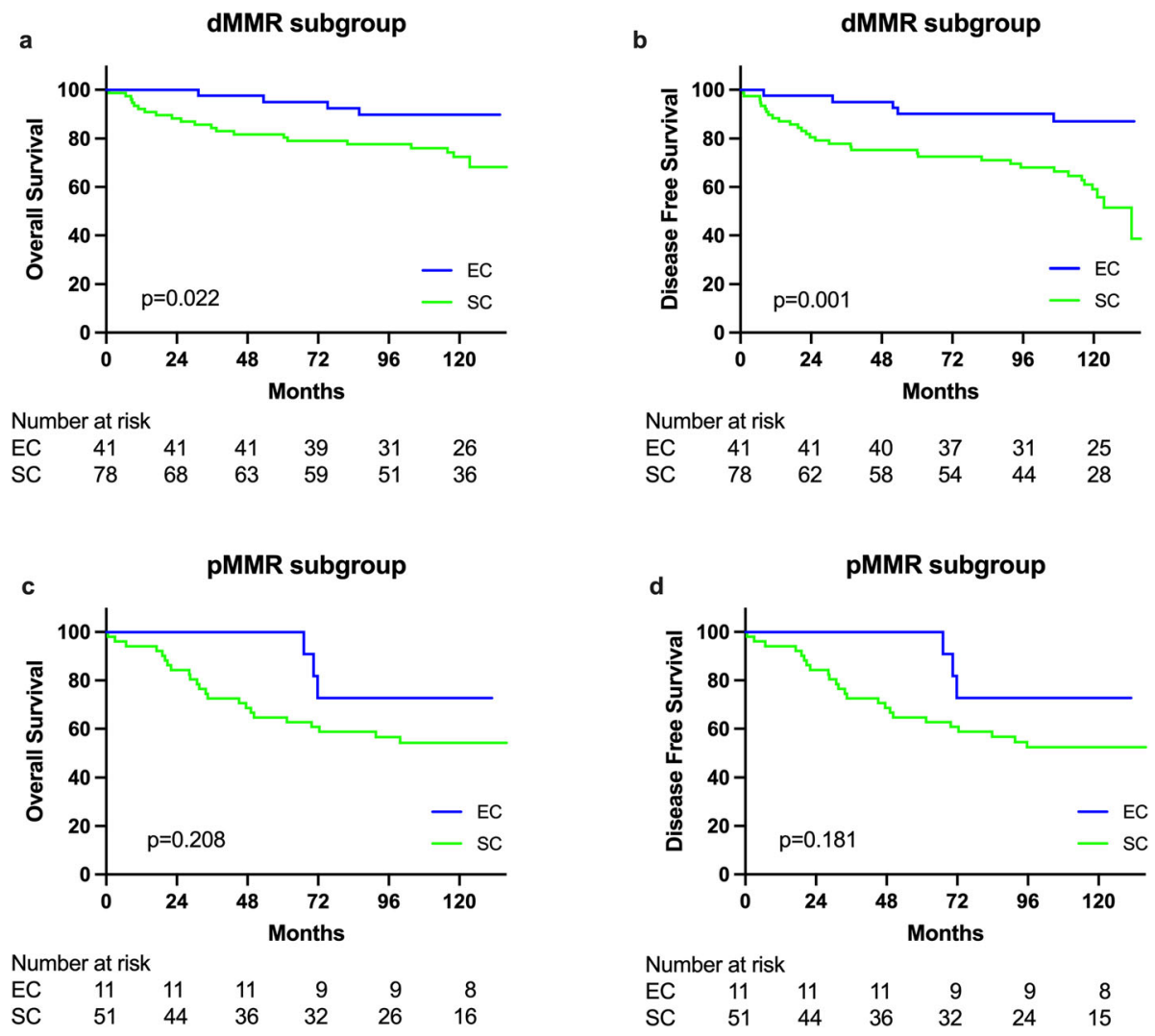

Fig. 4 Comparison of overall survival (a) and disease-free survival (b) between patients in dMMR subgroup who had extended colectomy (EC) and those who had segmental colectomy (SC); Comparison of overall survival (c) and disease-free survival (d) between patients in pMMR subgroup who had extended colectomy (EC) and those who had segmental colectomy (SC)

follow up. However, we did not observe similar findings because in our study, 6 of $34(17.6 \%)$ patients with MSH2/ MSH6 loss and 5 of $81(6.2 \%)$ MLH1/PMS2 loss patients developed metachronous CRC. The difference may be resulted from different population and follow up durations.
As showing in our study and others, pMMR status is more likely in cases such as older age ( 54.6 years old in this study) $[4,16,18,20]$, left colon predominant $(30.8 \%$ in this study) $[4,16]$ and higher rate of rectum involved (35.4\% in this study) [4, 16-18]. In these cases, SC might

Table 7 Univariate and multivariate analyses of clinicopathological features with OS and DFS among 181 HNPCC patients who underwent curative resection for CRC, according to MMR status

\begin{tabular}{|c|c|c|c|c|c|c|c|}
\hline & \multirow[t]{2}{*}{ Characteristics } & \multicolumn{3}{|c|}{ Univariate } & \multicolumn{3}{|c|}{ Multivariate } \\
\hline & & $\overline{\mathrm{HR}}$ & $95 \% \mathrm{Cl}$ & $\overline{p \text { Value }}$ & $\overline{H R}$ & $95 \% \mathrm{Cl}$ & p Value \\
\hline \multirow[t]{2}{*}{ OS } & $\mathrm{dMMR+EC}$ & 1 & & & 1 & & \\
\hline & $\mathrm{dMMR}+\mathrm{SC}$ & 3.252 & $1.115-9.485$ & 0.031 & 2.387 & $0.788-7.229$ & 0.124 \\
\hline \multirow[t]{2}{*}{ DFS } & $\mathrm{dMMR+EC}$ & 1 & & & 1 & & \\
\hline & $\mathrm{dMMR}+\mathrm{SC}$ & 4.252 & $1.653-10.935$ & 0.003 & 3.526 & $1.346-9.236$ & 0.010 \\
\hline \multirow[t]{2}{*}{ OS } & $\mathrm{pMMR+EC}$ & 1 & & & 1 & & \\
\hline & pMMR+SC & 2.128 & $0.638-7.098$ & 0.219 & 1.919 & $0.532-6.918$ & 0.319 \\
\hline \multirow[t]{2}{*}{ DFS } & $\mathrm{pMMR+EC}$ & 1 & & & 1 & & \\
\hline & $\mathrm{pMMR}+\mathrm{SC}$ & 2.219 & $0.667-7.377$ & 0.193 & 1.991 & $0.555-7.140$ & 0.291 \\
\hline
\end{tabular}


be considered if MMR status or genetic testing data not available. However, it is highly recommended that EC to be considered as an option in HNPCC patients with right colon tumor because of its higher rate of dMMR status or Lynch syndrome.

Age and comorbidities are two other factors should be concerned when we make EC as choice of operation, because EC had higher rate of postoperative complication and poor life of quality of these patients. In these cases, a proportion of dMMR patients may resulted from hyper-methylation of MMR genes rather than gene mutations [22]. In other words, aging patients may not be the case of lynch syndrome, moreover, there is fewer life expectancy in elderly to develop metachronous CRC. So, $\mathrm{SC}$ followed by close observation seems enough. However, as presented in our study, we combined family history of AC-II determined HNPCC patients and MMR status and found the average age of onset is below 50 years-old. Comorbidities are thus rare in these age group. The rate of metachronous cancer may vary from the aggressiveness of colonoscopy surveillance and the length of follow up period [23]. Moreover, AC-II positive patients with pMMR status were reported as another subgroup of familiar hereditary cancer such as FCCTX and showing relative lower rate of metachronous CRC compared with lynch syndrome $[4,16-18,20]$. We previously reported the cumulative rate of metachronous cancer in another study is 12 and $2.7 \%$ respectively for dMMR and pMMR HNPCC patients at 10 years, showing a relatively lower rate compared with previous studies showing $20 \%$ at 10 years follow up [21]. The difference of rate may be related to our aggressive postoperative surveillance strategy and metachronous cancer rate at 10 years may up to $40 \%$ in different population such as Lynch syndrome patient group [24].

This was a retrospective study, we included a clinical defined HNPCC group and the surveillance interval varied from one to three years depending on various physicians taking care policies. However, in dMMR/HNPCC patients, SC followed with surveillance strategy showed worse DFS in this study. Therefor how strict colonoscopy surveillance we should offer remained unclear for such patients because Lynch syndrome cancer was considered with a more rapid growth rate than sporadic type CRC.

In clinical setting, if young patients with small kindreds or unreliable family histories, it was difficult to apply our proposed strategy because our study based on combination of AC-II criteria and MMR status. Regarding universal MMR screening it may include some proportion of patients who resulted from hypermethlation of MMR genes not fit the case we discussed here. However, for all clinically defined HNPCC patient post-operation MMR status should be routinely checked. Because post- operative colonoscopy and extra-colonic surveillance were thus individualized based on MMR data [25]. Postoperation stringent colonoscopy surveillance of dMMR patients underwent segmental resection was recommended because metachronous CRC risk becomes an important issue while colonoscopy follow up for pMMR patients underwent segmental resection may similar to sporadic CRC because their low incidence of metachronous CRC [26]. The incidence of metachronous CRC is determined by the type of CRC and the length of follow up. In this study, only 11 patients developed metachronous CRC because of the short follow up period and inclusion of limited cases. As we reported previously [27] the frequencies of metachronous CRC in patients with sporadic CRC and HNPCC are 2.36 and 7.55 per 1000 person-year, respectively [27].

Although HNPCC patients defined by AC-II criteria clinically, we presented a different phenotypes between dMMR and pMMR subgroups in this study and it is consisted with previous literatures $[4,16-17,20]$. That right colon predominance $(61.2 \%$ V.S. $33.8 \%)$ in dMMR patients while left colon (30.8\% V.S. 24.8\%) and rectal tumor predominance (35.4\% V.S. $14.0 \%)$ in pMMR patients. The left colon and rectum predominant in pMMR patients contributed to significantly less frequency of extended colectomy (procto-colectomy or total colectomy) performed for the index tumor located at rectum or left than index tumor at right colon.

This study is limited by its retrospective nature. Although it comes from a cohort of patients with standardized data collection, providing the opportunity to compare dMMR and pMMR subgroups in HNPCC patients in terms of clinicopathologic factors and surgical outcomes, it did not answer if extra-colonic cancer can be prevented by prophylactic surgery such prophylactic hysterectomy because we have only limited cases with dMMR status developed endometrial cancers. It should also be noted that it is possible, given the multiple statistical comparisons performed in this analysis, that the noted associations could be chance findings.

\section{Conclusions}

Among HNPCC patients, distinct clinic-pathological features existed between dMMR and pMMR subgroups. Significantly better overall survival, younger age of onset and higher rate of metachronous CRC exist in dMMR subgroup comparing with pMMR subgroup.

Although there are no survival difference in EC and SC groups in dMMR patients, EC significantly improved DFS and was thus recommended for dMMR subgroup but not for pMMR subgroup in HNPCC patients. Preoperative tumor MMR status is highly recommended for help making decision of operation types (EC or SC). 


\section{Abbreviations}

HNPCC: Hereditary nonpolyposis colorectal cancer; EC: Extended colectomy; SC: Segmental colectomy; CRC: Colorectal cancer; IHC: Immunohistochemical; MMR: Mismatch repair; pMMR: proficient mismatch repair; dMMR: deficient mismatch repair; LS: Lynch syndrome

\section{Acknowledgements}

The authors would like to thank the Chang Gung Memorial Hospital Lin-kow mainbranch for partial financial support of this research and Grant number: CMRPG3J0392.

\section{Conflict of interest}

All the authors do not accept any financial or other interest that is relevant to the subject matter under consideration in this article.

\section{Authors' contributions}

Concept and design: Jy-Ming Chiang, Chun-Kai Liao.. Provision of study patients:. Jy-Ming Chiang, Chun-Kai Liao. Collection and assembly of data: JyMing Chiang, Chun-Kai Liao, Yueh-Chen Lin, Yu-Jen Hsu. Data analysis and interpretation: Jy-Ming Chiang, Chun-Kai Liao, Yih-Jong Chern and Jeng-Fu You. Manuscript writing: Jy-Ming Chiang, Chun-Kai Liao. Final approval of manuscript: Jy-Ming Chiang, Chun-Kai Liao,. All authors read and approved the final manuscript

\section{Funding}

This study was not funded by any outside source.

\section{Availability of data and materials}

Not applicable.

\section{Declarations}

\section{Ethics approval and consent to participate}

The study was approved by the IRB of Chang Gung Memorial Hospital (IRB201801201B)

Consent for publication: Not applicable.

\section{Competing interests}

The authors declare that they have no competing interests.

\section{Received: 24 March 2021 Accepted: 9 June 2021}

Published online: 29 June 2021

\section{References}

1. Parry S, Win AK, Parry B, Macrae FA, Gurrin LC, Church JM, et al. Metachronous colorectal cancer risk for mismatch repair gene mutation carriers: the advantage of more extensive colon surgery. Gut. 2011;60(7): 950-7.

2. Lynch HT, Smyrk T, Lynch JF. Overview of natural history, pathology, molecular genetics and management of HNPCC (Lynch syndrome). Int J Cancer. 1996;69(1):38-43.

3. de Vos tot Nederveen Cappel WH. Surveillance for hereditary nonpolyposis colorectal cancer: a long-term study on 114 families. Dis Colon Rectum. 2002;45(12):1588-94. https://doi.org/10.1007/s10350-004-7244-3.

4. Valle L, Perea J, Carbonell P, Fernandez V, Dotor AM, Benitez J, et al. Clinicopathologic and pedigree differences in Amsterdam-I positive hereditary nonpolyposis colorectal cancer families according to tumor microsatellite instability status. J Clin Oncol. 2007;25(7):781-6. https://doi. org/10.1200/JCO.2006.06.9781.

5. Son IT, Kim D-W, Jeong S-Y, Shin Y-K, Ihn MH, Heung-Kwon O, et al. Clinicopathological Features and Type of Surgery for Lynch Syndrome: Changes during the past two decades. Cancer Res Treat. 2016;48(2):605-11.

6. de Vos tot Nederveen Cappel WH, Buskens E, van Duijvendijk P, et al. Decision analysis in the surgical treatment of colorectal cancer due to a mismatch repair gene defect. Early-Onset Colon Cancer. 2003;52:1752-5.

7. Win AK, Parry S, Parry B, Kalady MF, Macrae FA, Ahnen DJ, et al. Risk of metachronous colon cancer following surgery for rectal cancer in mismatch repair gene mutation carriers. Ann Surg Oncol. 2013;20(6):1829-36. https:// doi.org/10.1245/s10434-012-2858-5.
8. Rodriguez-Bigas MA, Moeslein G. Surgical treatment of hereditary nonpolyposis colorectal cancer (HNPCC, Lynch syndrome). Familial Cancer. 2013;12(2):295-300. https://doi.org/10.1007/s10689-013-9626-y.

9. Syngal S, Brand RE, Church JM, Giardiello FM, Hampel HL, Burt RW, et al. ACG clinical guideline: genetic testing and management of hereditary gastrointestinal cancer syndromes. Am J Gastroenterol. 2015;110(2):223-62. https://doi.org/10.1038/ajg.2014.435.

10. Kalady MF, McGannon E, Vogel JD, Manilich E, Fazio WW, Church JM. Risk of colorectal adenoma and carcinoma after colectomy for colorectal cancer in patients meeting Amsterdam criteria. Ann Surg. 2010;252(3):507-511; discussion 511-3. doi:

11. Herzig DO, Buie WD, Weiser MR, You YN, Rafferty JF, Feingold D, Steele SR. Clinical practice guidelines for the surgical treatment of patients with Lynch syndrome. Dis Colon Rectum 2017:60(2):137-143. doi: https://doi.org/10.1 097/DCR.0000000000000785. PMID: 28059909.

12. Heneghan HM, Martin ST, Winter DC. Segmental vs extended colectomy in the management of hereditary nonpolyposis colorectal cancer: a systematic review and meta-analysis. Color Dis 2015;17(5):382-389. doi: https://doi. org/10.1111/codi.12868. PMID: 25510173.

13. Haanstra JF, de Vos Tot Nederveen Cappel WH, Gopie JP, Vecht J, Vanhoutvin SA, Cats A, et al. quality of life after surgery for colon cancer in patients with Lynch syndrome: partial versus subtotal colectomy. Dis Colon Rectum. 2012;55(6):653-9. https://doi.org/10.1097/DCR.0b013e31824f5392.

14. Colling R, Church DN, Carmichael J, Murphy L, East J, Risby P, Kerr R, Chetty $R$, Wang LM. Screening for Lynch syndrome and referral to clinical genetics by selective mismatch repair protein immunohistochemistry testing: an audit and cost analysis. J Clin Pathol 2015;68(12):1036-1039. doi: https://doi. org/10.1136/jclinpath-2015-203083. Epub 2015 Jul 22. PMID: 26201544.

15. David Tougeron, Guillaume Mouillet, Isabelle Trouilloud, Thierry Lecomte, Romain Coriat, Thomas Aparicio, Gaetan Des Guetz, Cédric Lécaille, Pascal Artru, Gaelle Sickersen, Estelle Cauchin, David Sefrioui, Tarek Boussaha, Aurélie Ferru, Tamara Matysiak-Budnik, Christine Silvain, Lucie KarayanTapon, Jean-Christophe Pagès, Dewi Vernerey, Franck Bonnetain, Pierre Michel, Julien Taïeb, Aziz Zaanan, Efficacy of Adjuvant Chemotherapy in Colon Cancer With Microsatellite Instability: A Large Multicenter AGEO Study, JNCl, Volume 108, Issue 7, July 2016, djv438, https://doi.org/10.1093/ jnci/djv438

16. Shiovitz S, Copeland WK, Passarelli MN, Burnett-Hartman AN, Grady WM, Potter JD, et al. Characterisation of familial colorectal cancer Type X, Lynch syndrome, and non-familial colorectal cancer. Br J Cancer. 2014;111(3):598-602.

17. Llor X, Pons E, Xicola R, et al. Differential features of colorectal cancers fulfilling Amsterdam criteria without involvement of the mutation pathway. Clin Cancer Res. 2005;11(20):7304-10. https://doi.org/10.1158/1078-0432. CCR-05-0965.

18. Tatsuro Yamaguchi, Yoichi Furukawa, Yusuke Nakamura, Nagahide Matsubara, Hideki Ishikawa, Masami Arai, Naohiro Tomita, Kazuo Tamura, Kokichi Sugano, Chikashi Ishioka. Comparison of clinical features between suspected familial colorectal cancer type $X$ and Lynch syndrome in Japanese patients with colorectal cancer: a cross-sectional study conducted by the Japanese Society for Cancer of the Colon and Rectum Japanese J Clin Oncol, Volume 45, Issue 2, 1 February 2015, Pages 153-159,

19. Chen JR, Chiang JM, Changchien CR, Chen JS, Tang RP, Wang JY. Mismatch repair protein expression in Amsterdam II criteria-positive patients in Taiwan. Br J Surg 2008;95(1):102-110. doi: https://doi.org/10.1002/bjs.5786. PMID: 18064717.

20. Mueller-Koch $Y$, Vogelsang $H$, Kopp R, Lohse P, Keller G, Aust D, Muders M, Gross M, Daum J, Schiemann U, Grabowski M, Scholz M, Kerker B, Becker I, Henke G, Holinski-Feder E. Hereditary non-polyposis colorectal cancer: clinical and molecular evidence for a new entity of hereditary colorectal cancer. Gut. 2005;54(12):1733-1740. doi: https://doi.org/10.1136/gut.2004. 060905. Epub 2005 Jun 14. PMID: 15955785; PMCID: PMC1774771.

21. Quezada-Diaz FF, Hameed I, von Mueffling A, Salo-Mullen EE, Catalano JD, Smith JJ, Weiser MR, Garcia-Aguilar J, Stadler ZK, Guillem JG. Risk of Metachronous Colorectal Neoplasm after a Segmental Colectomy in Lynch Syndrome Patients According to Mismatch Repair Gene Status. J Am Coll Surg. 2020 Apr;230(4):669675. doi: https://doi.org/10.1016/j.jamcollsurg.2020.01.005. Epub 2020 Jan 30. PMID: 32007537; PMCID: PMC7104918.

22. Thiel A, Heinonen M, Kantonen J, Gylling A, Lahtinen L, Korhonen M, Kytölä S, Mecklin JP, Orpana A, Peltomäki P, Ristimäki A. BRAF mutation in sporadic colorectal cancer and Lynch syndrome. 
Virchows Arch 2013;463(5):613-621. doi: https://doi.org/10.1007/ s00428-013-1470-9. Epub 2013 Aug 21. PMID: 23963522.

23. Erenay FS, Alagoz O, Banerjee R, Said A, Cima RR. Cost-effectiveness of alternative colonoscopy surveillance strategies to mitigate metachronous colorectal cancer incidence. Cancer. 2016;122(16):2560-70. https://doi.org/1 0.1002/cncr.30091

24. Lynch HT, Watson P, Lanspa SJ, Marcus J, Smyrk T, Fitzgibbons RJ Jr, Kriegler M, Lynch JF. Natural history of colorectal cancer in hereditary nonpolyposis colorectal cancer (Lynch syndromes I and II). Dis Colon Rectum 1988;31(6): 439-444. doi: https://doi.org/10.1007/BF02552613. PMID: 3378468.

25. Stjepanovic N, Moreira L, Carneiro F, Balaguer F, Cervantes A, Balmaña J, Martinelli E; ESMO guidelines committee. Electronic address: clinica Iguidelines@esmo.org. Hereditary gastrointestinal cancers: ESMO clinical practice guidelines for diagnosis, treatment and follow-upt. Ann Oncol 2019;30(10):1558-1571. doi: https://doi.org/10.1093/annonc/mdz233. PMID: 31378807.

26. Karlitz JJ, Sherrill MR, DiGiacomo DV, Hsieh MC, Schmidt B, Wu XC, Chen WW. Factors Associated With the Performance of Extended Colonic Resection vs. Segmental Resection in Early-Onset Colorectal Cancer: A Population-Based Study. Clin Transl Gastroenterol. 2016;7(4):e163. doi: https://doi.org/10.1038/ctg.2016.17. PMID: 27077958; PMCID: PMC4855160.

27. Po-Yen Lin PY, Chiang JM, Huang HY, You JF, Chiang SF, Hsieh PS, et al. Various clinicopathological features of patients with metachronous colorectal cancer in relation to different diagnostic intervals. Int J Color Dis. 2018;33(9):1235-40. https://doi.org/10.1007/s00384-018-3106-7.

\section{Publisher's Note}

Springer Nature remains neutral with regard to jurisdictional claims in published maps and institutional affiliations.

Ready to submit your research? Choose BMC and benefit from:

- fast, convenient online submission

- thorough peer review by experienced researchers in your field

- rapid publication on acceptance

- support for research data, including large and complex data types

- gold Open Access which fosters wider collaboration and increased citations

- maximum visibility for your research: over $100 \mathrm{M}$ website views per year

At $\mathrm{BMC}$, research is always in progress.

Learn more biomedcentral.com/submissions 